\title{
Tres ciclos migratorios en Chiapas: interno, regional e internacional
}

\author{
DANIEL VILLAFUERTE SOLÍS* \\ MARÍA DEL CARMEN GARCÍA AGUILAR**
}

RESUMEN: Este artículo describe y analiza el fenómeno migratorio chiapaneco en tres momentos a partir de los cambios ocurridos en la economía interna, regional e internacional. La hipótesis que subyace en el trabajo es que la migración internacional de chiapanecos a Estados Unidos forma parte de un proceso cíclico que inició con la migración interna, luego pasó a la migración interestatal para, finalmente, dar paso a la migración internacional que presupone una acumulación intergeneracional de experiencias migratorias que han dado como resultado la formación de redes migratorias. La migración a Estados Unidos está ligada a la irrupción del modelo neoliberal y sus políticas que profundizaron la crisis de la producción rural, la lucha agraria y la pobreza, que articuladas a otros fenómenos como el levantamiento zapatista del 1 de enero de 1994 y los efectos del cambio climático, produjeron la sistemática migración que confluyó con los flujos transfronterizos centroamericanos.

PALABRAS CLAVE: Chiapas, migración, pobreza, crisis, flujos transfronterizos.

\footnotetext{
* Investigador titular del Centro de Estudios Superiores de México y Centroamérica de la Universidad de Ciencias y Artes de Chiapas, México.

**Investigadora-docente del Centro de Estudios Superiores de México y Centroamérica de la Universidad de Ciencias y Artes de Chiapas, México.
} 
ABSTRACT: This article describes and analyzes the Chiapas migratory phenomenon in three stages entrenched in the transformations operated in the domestic, regional and international economy. The hypothesis underlying this paper is that the international migration process from Chiapas to the United States is part of a cyclical process that began with internal migration, transited to inter-state migration, and finally gave rise to the international migration through an intergenerational accumulation of cross-border experiences and the formation of migratory networks. This process is associated to the imposition of the neoliberal model and its related policies, which have excavated the crisis of rural production, the upsurge of the agrarian struggle, and the expansion of poverty; all this in close interrelation with other phenomena such as the Zapatista uprising in January 1, 1994, the impacts of climate change in the region, and its convergence with the rise of crossborder Central American migration flows.

KEY WORDS: Chiapas, migration, poverty, crises, cross-border flows. 


\section{INTRODUCCIÓN}

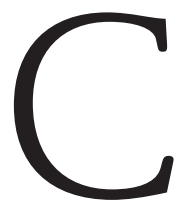

hiapas es el estado más fronterizo del sur de México; comparte con Guatemala una frontera de más de 800 km. En 2005 era una de las entidades del país con mayor número de habitantes: con 4 millones 293 mil 459, ocupaba el séptimo lugar, por arriba de Nuevo León, Michoacán y Oaxaca (Inegi, 2008). De acuerdo con el Censo de Población y Vivienda de 2010, en Chiapas la población había aumentado a 4 millones 796 mil 580, es decir, más de medio millón de personas; según la misma fuente, el número de hablantes de lengua indígena de 5 años y más alcanzó un millón 177 mil 432, lo que representa 17.6\% de hablantes de lengua indígena en el país y $24.5 \%$ en el estado, porcentajes que podrían incrementarse al contabilizar los grupos minoritarios de otras lenguas, estos indicadores dan cuenta de la diversidad y complejidad de la realidad social de Chiapas y dificultan la comprensión del fenómeno migratorio.

Por su extensión territorial, Chiapas es el estado número diez al disponer de $73,289 \mathrm{~km}^{2}$. La densidad de población de 58 habitantes por $\mathrm{km}^{2}$ se ubica por arriba de la media nacional. Sin embargo, es la entidad que acusa los mayores rezagos sociales y el menor grado de desarrollo económico. Presenta el grado de marginación social más alto del país y los niveles de pobreza alcanzan proporciones equiparables a la de algunos países de África subsahariana: $78.4 \%$ de la población chiapaneca se encuentra en condiciones de pobreza y $32.8 \%$ en pobreza extrema; $82.4 \%$ carece de seguridad social; $43.2 \%$ carece de acceso a los servicios de salud y $30.3 \%$ padece hambre (Coneval, 2010). Chiapas contribuye al grupo de los 100 municipios más pobres y marginados del país; en este listado se localizan los municipios que cuentan con una población mayoritariamente indígena, entre los que destacan Aldama, Chalchihuitán, Chamula, Chanal, Huixtán, Chilón, Larraínzar, Mitontic, Oxchuc, Pantelhó, San Juan Cancuc, Santiago el Pinar y Sitalá. ${ }^{1}$

${ }^{1}$ Estos municipios están incorporados en el programa Cruzada Nacional contra el Hambre, véase: http://sinhambre.gob.mx/. 
Después de Oaxaca, Chiapas es el segundo estado con mayor población indígena. Este sector, que representa poco más de $25 \%$ de la población chiapaneca, es la más pobre y la que menos acceso tiene a los servicios básicos: luz eléctrica, agua potable, drenaje, educación y salud. En las localidades rurales e indígenas, la tasa de mortalidad infantil es de 75 por cada 1,000 menores. Por otra parte, según la información del Centro Nacional para la Prevención y Control del VIH/SIDA (2012), el número de infectados con VIH en el estado ha crecido considerablemente: del onceavo lugar nacional que tenía en 2001 pasó al sexto en 2012, con 7,189 casos acumulados; en el caso de las mujeres ocupó el quinto lugar con 1,925 casos. Habría que investigar cuántos de estos se relacionaron con la migración, pues el mayor incremento coincide con el periodo 2002-2012, una década de fuerte migración en el que se registraron 5,405 casos.

Chiapas representa el rostro más visible de la pobreza y la marginación en el México posterior a la entrada en vigor del Tratado de Libre Comercio de América del Norte (TLCAN). En contraposición se encuentra, en el norte de México, Baja California, una entidad que comparte frontera con el estado de California, Estados Unidos, cuyos indicadores de rezago social distan enormemente de los de Chiapas: con casi tres millones de habitantes, sólo el 9.2\% de su población tiene pobreza de patrimonio; $2.3 \%$, pobreza de capacidades, y $1.3 \%$, pobreza alimentaria. Las distancias geográficas y sociales son enormes, uno en el extremo sur y otro en el extremo norte del territorio mexicano, uno comparte frontera con Guatemala y el otro con Estados Unidos. ¿̇Se puede establecer alguna relación entre pobreza y marginación con la vecindad?

La debilidad estructural de la economía se refleja en la distribución de la población económicamente activa (PEA) y la generación del producto interno bruto (PIB): al último trimestre de 2012, según la Encuesta Nacional de Ocupación y Empleo (sTPS, 2012), de la población ocupada, 42.6\% correspondió al sector psrimario, $12.5 \%$ al secundario y $44.7 \%$ al terciario. Sin embargo, el primario escasamente genera el 10\% del PIB, mientras que el terciario contribuye con más del $64 \%$. Además, en el campo conviven dos estructuras productivas: por una parte, una agricultura campesina minifun-

$6 \frac{\text { PRIMER SEMESTRE } 2014}{\text { MIGRACIÓN Y DESARROLLO NÚM. } 22}$ 
dista, con serias dificultades para producir alimentos básicos que no garantizan la reproducción biológica y social de las familias y, por otra, una actividad agropecuaria poco eficiente en el uso y manejo de los recursos productivos que se traduce en un patrón productivo tradicional, extensivo, poco diversificado e incapaz de favorecer el empleo de una mano de obra creciente que se genera en el sector social de la agricultura.

En este marco, producto de un largo proceso histórico, tiene cabida un fenómeno de singular importancia que ha venido creciendo en escasas dos décadas. Se trata de la migración a Estados Unidos. Una migración que incluye, sobre todo, a indígenas y campesinos, en menor medida a sectores medios y pobres de centros urbanos que han sido afectados por el modelo económico neoliberal. Pensamos que este fenómeno que comienza en la década de 1990 no puede explicarse sólo por la crisis económica de los últimos años. Si bien la situación económica actual es la principal responsable de los movimientos de población, es necesario considerar la configuración de estructuras económicas, sociales y políticas que dieron lugar a una dinámica migratoria que fue pasando de una fase a otra: del ciclo de las migraciones internas a las interestatales y de éstas a la actual de carácter internacional, mediada por una reorientación ascendente de las migraciones de chiapanecos a los estados del sur de México hacia a las entidades de la frontera norte.

Primer ciclo: las migraciones internas

Chiapas ha sido un estado de importantes movimientos poblacionales. Sin embargo, hasta bien entrado el siglo xx, las migraciones habían sido de carácter interno, generadas por dos factores: 1) la existencia de una estructura agraria altamente concentrada, y 2) la consolidación de una economía de plantaciones que demandaba una creciente mano de obra. Estos componentes tienen un peso significativo en la formación económico-social regional caracterizada tanto por la desigualdad en la dotación de recursos productivos — principalmente la tierra- como por la especialización productiva. 
El contexto de los mercados nacional e internacional va generando una división regional del trabajo: la región del Soconusco se caracteriza por una estructura productiva de plantaciones, favorecida en buena medida por la política de colonización extranjera durante el régimen de Porfirio Díaz; ${ }^{2}$ la región de la Costa se especializa en ganadería bovina y agricultura comercial; la región Norte y la Selva Lacandona se distinguen por la producción de maderas preciosas, café, cacao, maíz, ganado bovino y porcino; la región de Los Altos, importante por su población indígena, con una estructura agraria y agrícola bastante precaria, se destaca por la producción y reproducción de mano de obra barata que demandan otras regiones de Chiapas, como el Soconusco; finalmente, la región de los Valles Centrales se especializa en la producción de ganado bovino y granos básicos, elementos fundamentales para la reproducción del capital.

La especialización productiva que ocurre en Chiapas profundiza la dependencia con respecto a los mercados nacional e internacional y permite la reproducción de las desigualdades sociales. Es el caso de las plantaciones cafetaleras que requieren de un gran «ejército de jornaleros», lo cual es posible gracias al desarrollo desigual generado por la propia estructura agraria y la lógica de las relaciones capitalistas que se van extendiendo en todo el territorio chiapaneco. De esta manera, regiones como Los Altos, con población mayoritariamente indígena, se convierten en verdaderas fábricas de peones asalariados, y además con la creación de mecanismos como el sistema de enganche -institucionalizado en 1937, durante la administración del presidente Lázaro Cárdenas, mediante la creación de la Dirección de Asuntos Indígenas y del Sindicato de Trabajadores Indígenas-, se aseguraba el abastecimiento de fuerza de trabajo en cada ciclo agrícola (Morales et al., 1978; Fernández y Wasserstrom, 1977; Wasserstrom, 1980).

2 «Hacia 1894-1895 la compañía inglesa-mexicana Land and Colonization Chiapas Ltd se hizo cargo del negocio de tierras en el Soconusco e inmediatamente elevó el precio de 50 centavos de dólares el acre a 1 y 1.50 dólares. La citada compañía era, sin duda, una de las más agresivas en su ramo, (...) bajo el amparo del gobierno de Díaz se apropió de 2 millones de hectáreas en Chiapas, 600 mil de ellas en los partidos de Tonalá y Soconusco» (Báez, 1985: 157).

MIGRACIÓN Y DESARROLLO NÚM. 22 
La expansión de la frontera cafetalera se convirtió en un poderoso factor de atracción de mano de obra de los municipios alteños a las fincas del Soconusco. En menos de cuatro décadas la superficie sembrada de café se multiplicó casi por cuatro al pasar de 7 mil a 27 mil hectáreas en el periodo de 1908 a 1946. Báez refiere que:

[...] de las 94 fincas registradas en el Soconusco a fines de los años treinta, 69 estaban en manos extranjeras, particularmente de alemanes. La actividad no sólo la controlaban en el cultivo, sino en el beneficiado y su exportación. Las fincas alemanas producían más de 100 mil quintales-oro y tenían en su poder más de 10 mil hectáreas, de las 60 mil que abarcaba toda la franja cafetalera, incluyendo terrenos de bosques, potreros y monte (1985: 168).

El floreciente desarrollo capitalista en la región del Soconusco a partir de las plantaciones agrícolas, en particular de la expansión de la frontera cafetalera, trajo consigo una verdadera explosión demográfica. «Soconusco empezó a cambiar tanto física como estructuralmente. La población aumentó de 11,218 en 1883, cuando la economía regional languidecía, a 28,907 habitantes en 1895, y a 36,000 a principios de siglo (Spencer, 1988, citado por Villafuerte, 2000: 142). En esta misma línea, Viqueira (2008: 295) refiere que, «junto con el café, la población del Soconusco tuvo un crecimiento espectacular: en 50 años se multiplicó por más de cinco veces, pasando de más de 17,000 habitantes en 1880 a más de 97,000 en 1930».

Quienes más aportaron al desarrollo de la cafeticultura del Soconusco fueron los migrantes indígenas de Los Altos de Chiapas. Los archivos de la Subsecretaría de Asuntos Indígenas (hoy Secretaría de Pueblos Indios), reportan un promedio de 11,601 jornaleros al año hacia las fincas cafetaleras, durante el periodo 1979-1986. A esta cifra debemos sumar una cantidad similar que se contrataba directamente, sin intermediación del enganchador, con lo cual la cifra ascendería entre 30 y 35 mil jornaleros por año. Un dato que apoya esta idea es aportado por la mencionada Subsecretaría, que en 1979 reporta la contratación de 15,329 jornaleros. Durante este periodo la migración de la población indígena chiapaneca a las fincas cafetaleras 
comenzaba a ser reemplazada por la de jornaleros provenientes del altiplano guatemalteco, por ejemplo «el número de jornaleros guatemaltecos documentados en las garitas de Ciudad Hidalgo y Unión Juárez pasó de 37,848 en 1984 a 60,944 en 1992» (Martínez, 1994: 125).

El otro factor de migración interna fue el problema agrario, particularmente en las tierras altas. La población de las regiones Sierra y Altos de Chiapas se reproducía en minúsculas parcelas por lo que requería de ingresos complementarios a través de la venta de mano de obra a las fincas cafetaleras, sin perder de vista la posibilidad de conseguir tierras en otras regiones del estado. Es así que desde mediados de los sesenta se inicia un proceso de migración espontánea y dirigida con miras a colonizar la Selva Lacandona. Eran los años de la conquista del trópico que resolvería — se decía - la presión sobre una estructura agraria altamente concentrada y garantizaría la autosuficiencia alimentaria.

El movimiento campesino que cobra fuerza a mediados de los años setenta expresa la primera gran crisis de una estructura agraria atrasada y rentista que sostenía a una economía de plantaciones y una ganadería bovina extensiva, que ocupa grandes superficies de tierra y genera muy poco empleo, en medio de una pobreza de amplios sectores de la población chiapaneca, principalmente campesinos e indígenas. ${ }^{3}$

Esto explica también la creciente oleada de migraciones hacia la Selva Lacandona, ya sea de manera espontánea o dirigida por la política agraria del momento. En el Reporte Final de Actividades del Fideicomiso de la Selva Lacandona (CIES, 1977) podemos encontrar una tesis fundamental, que coincide con nuestro punto de partida:

La problemática socioeconómica de Chiapas se centra básicamente en un amplio fenómeno de polarización (distanciamiento y, frecuentemente, contradicción económica y social). Esta polarización, que se tipifica en la contradicción entre la población y recursos (amplios grupos de población pauperizada y regiones ricas de poca densidad, donde, además, un pequeño grupo dispone de una gran

${ }^{3}$ Esta problemática ha sido ampliamente documentada por Fernández y Tarrío (1983), Villafuerte, García y Meza (1997).

$10 \frac{\text { PRIMER SEMESTRE } 2014}{\text { MIGRACIÓN Y DESARROLLO NÚM. } 22}$ 
cantidad de recursos), tiene sus manifestaciones más características en las diferencias de ingreso, en la apropiación diferenciada del espacio productivo del estado, en la contradicción entre bienestar campesino y producción de excedentes, especialmente ganaderos, $y$, en general en la existencia profundamente conflictiva, de un sector de agricultura capitalista y otro de economía campesina, vinculados por relaciones de fuerte explotación del segundo por el primero (CIES, 1977).

Este documento hace revelaciones importantes que apoyan la tesis sobre el fenómeno de la polarización social-agraria en Chiapas y explican en buena medida los movimientos de la población:

En esta región —La Serranía Central—, de más de 300 mil habitantes en su mayoría indígenas (tzotziles, tzeltales, choles y tojolabales), los promedios de tierra son mínimos y la tierra es de mala calidad. La productividad de estos municipios es muy baja. En 1970 seis municipios de Los Altos produjeron el 1.1\% del valor de la producción agrícola del estado, mientras que el municipio de Tapachula por sí solo produjo el $10 \%$.

Pero la conquista del trópico se vio acompañada no sólo de colonización campesina sino también de la expansión de la ganadería bovina que disputó el uso del espacio productivo a los campesinos que ya vivían o que llegaron a colonizar. Un pasaje del texto citado que nos parece revelador de este proceso es el siguiente:

Las áreas boscosas de Tecpatán, donde se construyó la presa Malpaso y en las que más de 10,000 habitantes, antiguos pobladores y colonizadores espontáneos, trabajaban y convivían, y seguía existiendo la selva. En poco más de 10 años (de 1960 a 1972) el número de cabezas de ganado pasó de algo menos de 6,000 a 50,000 y, entonces sí, la selva desapareció y los campesinos comenzaron a experimentar escasez de tierra, agotamiento de la capacidad de las áreas rurales del municipio para recibir más campesinos. Tecpatán estaba siendo un área de inmigración de tzotziles y comenzando un proceso de expulsión de campesinos hacia las selvas contiguas de Veracruz y Oaxaca. 
El entorno donde ocurren los movimientos internos de población es de un estado fundamentalmente rural, donde no hay opciones de empleo en otros sectores de la economía. En 1970, según las cifras del IX Censo de Población y Vivienda, la población total de Chiapas ascendía a 1'569,053 habitantes, distribuidos en 7,740 localidades. Más de 97 de las localidades contaban con menos de 1,000 habitantes y concentraban 57\% de la población. Otro grupo importante de localidades, que se situaban en el rango de entre 1,000 y menos de 10,000 habitantes, que significaba $2.65 \%$, concentraba $26.8 \%$ de la población chiapaneca. En el polo opuesto, sólo 11 localidades de 10,000 habitantes y más, que constituían solamente $0.12 \%$ de las localidades, agrupaban $16.2 \%$ de la población. Es decir, cerca de $84 \%$ de la población vivía en localidades rurales menores de 10,000 habitantes, lo que explica en buena medida la enorme presión sobre la tierra.

Otro dato que debe tomarse en cuenta es la composición de la población por edades. A mediados del siglo xx, 72.4\% de la población era menor de 30 años, en 1960 este segmento de la población representó 73.2\% de la población chiapaneca y para 1970 ya constituía 74.3\% de la población. Esto da una idea de la enorme presión social sobre el recurso tierra, que se convierte en un recurso crítico para la supervivencia de miles de familias campesinas. En 1970, el Censo Agrícola Ganadero y Ejidal mostraba una realidad que poco había cambiado con el proceso revolucionario y con la administración de Cárdenas.

Por otra parte, de los 4’763,854 hectáreas censadas en 1970, el 56\% pertenecían a ejidos y comunidades. Sin embargo, si nos atenemos a los datos de la población descritos arriba veremos que la tierra por habitante disminuye considerablemente. En contraposición, en el sector privado encontramos una concentración evidente: de 28,119 predios en que se distribuye poco más de 2'960,000 hectáreas de propiedad privada, sólo 1.7\% de los predios concentraba $23.8 \%$ de la tierra, es decir, medio millón de hectáreas. Esta enorme cantidad de tierras en manos de 476 propietarios se dividían de la siguiente manera: 327 predios contaban con una superficie de entre 500 y 1000 hectáreas; 114 predios con una extensión de más de 1,000 y hasta 5,000 
hectáreas, y sólo 5 predios concentraban superficies mayores de 5,000 hectáreas (Villafuerte, 2001).

A este cuadro es necesario agregar el uso del suelo, donde poco más del $25 \%$ de las tierras censadas estaban ocupadas por la ganadería bovina, tanto en el sector privado como en el sector ejidal. Se trata de casi 649 mil hectáreas en el sector privado, sobre todo en el grupo con superficie mayor a 5 hectáreas, y de cerca de 555 mil hectáreas en el sector ejidal. Esto explica, en buena medida, la aparente paradoja donde prevalece una población rural y una PEA dedicada mayormente a las actividades primarias (72.8\%) frente a un PIB sectorial que sólo aporta alrededor de $30 \%$. Este patrón de ocupación del uso del suelo y de la mano de obra se reproduce en las décadas que siguen, situación que genera la profundización de los conflictos por la tierra y los procesos migratorios internos.

\section{CUADRO 1}

Tasa anual de migración neta en municipios seleccionados*

\begin{tabular}{lccc}
\hline \multicolumn{1}{c}{ MUNICIPIO } & $1940-1950$ & $1950-1960$ & $1960-1970$ \\
\hline Amatenango del Valle & -1.42 & -0.94 & -1.50 \\
Bochil & -2.18 & -0.98 & -1.68 \\
El Bosque & -0.66 & -1.39 & -1.23 \\
Chalchihuitán & +0.75 & -1.73 & -2.71 \\
Chamula & -0.69 & -0.84 & -0.99 \\
Chanal & -4.85 & +0.33 & -3.53 \\
Larraínzar & -2.03 & -3.65 & -1.91 \\
Mitontic & -0.91 & -0.59 & -6.24 \\
Pantepec & -0.50 & -2.38 & -0.78 \\
Pueblo Nuevo Solistahuacán & -0.82 & -1.57 & -0.17 \\
Rayón & -0.34 & -1.64 & -0.87 \\
Simojovel & -0.36 & -1.61 & -1.26 \\
Teopisca & +0.05 & -1.42 & -0.84 \\
Tumbalá & -0.88 & -1.31 & -2.82 \\
\hline
\end{tabular}

* La tasa que va de -0.59 y más se considera como un valor de fuerte expulsión. Fuente: Fideicomiso de la Selva Lacandona (1977).

En este marco podemos tener una mejor comprensión sobre los procesos migratorios internos de carácter estacional, básicamente de la región de 
Los Altos hacia la zona de central, también denominada Mesochiapas, y a la región Soconusco, y de forma definitiva hacia la Selva Lacandona, mediante procesos de colonización. En un horizonte de treinta años, que va de 1940 a 1970, podemos observar claramente una correlación directa entre la precariedad de recursos productivos de muchos municipios, principalmente de Los Altos, y la fuerte expulsión de población, tal como se presenta en el cuadro 1.

Amatenango del Valle, Chalchihuitán, Chamula, Chanal, Larraínzar, Mitontic y Teopisca pertenecen al selecto grupo de municipios de Los Altos de Chiapas con mayor tasa de expulsión de población. Se trata de municipios pequeños, con un problema estructural de recursos productivos. El conjunto de estos siete municipios suman un extensión territorial aproximada de 1,132.8 $\mathrm{km}^{2}$ y su población en 1940 ascendía a 40,505 habitantes, resultando una densidad de 35.7 habitantes por $\mathrm{km}^{2}$, una cifra muy superior a la media estatal que para ese año era de 9 habitantes por unidad de superficie. Hacia 1970 la población de estos municipios llegó a sumar 59,123 habitantes, con una densidad de 52.2 habitantes por $\mathrm{km}^{2}$, mientras que el promedio estatal se estableció en 20.

El municipio de Chamula es el que presenta la mayor densidad de población del grupo de municipios alteños al pasar de 195 habitantes por $\mathrm{km}^{2}$ en 1940 a 358 habitantes en 1970. En contraste, el municipio de Ocosingo, donde se ubica buena parte de la Selva Lacandona, y que fue objeto de colonización espontánea e inducida, poseía en 1940 un habitante por km², y para 1970 había crecido a 3 habitantes. En este último año, Chamula tenía una población muy cercana a la de Ocosingo: 29,789 frente 34,356. Además, es importante señalar que durante la década de los sesenta, la tasa de crecimiento de la población de Ocosingo fue de 5.8\%, muy por arriba del promedio del estado que se situó en $2.7 \%$. Este periodo coincide con la llegada de una gran cantidad de migrantes de Chiapas y de otros estados a las tierras selváticas de la Lacandona.

Estos datos concuerdan con las evaluaciones realizadas en campo por el equipo de investigación que participó en el Fideicomiso de la Selva Lacandona:

$14 \frac{\text { PRIMER SEMESTRE } 2014}{\text { MIGRACIÓN Y DESARROLLO NÚM. } 22}$ 
La zona suroccidental de la región Lacandona, zona de influencia de la ciudad de Comitán, constituye una región de fuerte inmigración a pesar de que no cuenta, como la zona norte de la selva, con vías de comunicación que la liguen con la red caminera del estado y a los centros estatales de importancia comercial, financiera y de servicios. La casi totalidad de los asentamientos de colonizadores cuentan sólo con senderos abiertos a machete que los comunican entre sí con distancias promedio de cuatro a cinco kilómetros, con un tiempo de una y media a dos horas de recorrido a pie.

La colonización de esta zona, hecha por tojolabales de la tierra templada de Margaritas, por tzeltales y tzotziles provenientes de Los Altos de Chiapas y de gente proveniente de los municipios cercanos a la frontera guatemalteca, desde la vertiente interna de la Sierra Madre hasta el municipio de La Independencia, data de la década de los cincuenta, pero se vio fuertemente incrementada en la década 1960-70 y en lo que va de los años setenta (CIES, 1977: 37).

En suma, la selva se convirtió en un gran espacio de frontera cuya ocupación avanzó rápidamente: las áreas forestales se fueron transformando en terrenos de cultivo y en áreas de pastizales para el ganado bovino. Paradójicamente, la ocupación del espacio, supuestamente vacío, comenzó a generar una serie de conflictos que hasta hoy no terminan de resolverse. Hacia mediados de los ochenta del siglo xx se estimaba una población de 140,000 habitantes, distribuidos como se expresa en el cuadro 2.

\section{CUADRO 2}

Distribución de la población en la región lacandona, estimaciones para 1985

\begin{tabular}{lccr}
\hline \multicolumn{1}{c}{ REGiones } & HABITANTES & \% DE POBLACIÓN & $\begin{array}{r}\text { SUPERFICIE } \\
\text { HECTÁREAS }\end{array}$ \\
\hline Reserva de Biosfera & - & - & 300,000 \\
Marqués de Comillas & 10,000 & 7.1 & 160,000 \\
Valles y Cañadas Centrales & 25,000 & 17.9 & 290,000 \\
Santo Domingo & 35,000 & 25.0 & 150,000 \\
Nuevos Centros de Población & 70,000 & 50.0 & 500,000 \\
\hline Región Lacandona & 140,000 & 100.0 & $1,400,000$ \\
\hline
\end{tabular}

Fuente: Mauricio, Valladares y García (1985). 
El principal protagonista de las tensiones en la Selva ha sido el Estado, que al intentar frenar el proceso de colonización, generó mayores problemas. Destaca una serie de resoluciones presidenciales sobre predios que ya estaban ocupados; en ello se inscribe el decreto presidencial de 1971 mediante el cual se dota a los lacandones una superficie comunal de más de $614 \mathrm{mil}$ hectáreas. A este respecto, el gobernador Manuel Velasco Suárez, en su segundo informe, refiere:

Menciono con emoción el acto agrario más grande y revolucionario, de contenido social reivindicatorio, que ampara la Nueva Ley de Reforma Agraria y al que Chiapas concurre con la expresión de profunda gratitud al régimen del Presidente Echeverría, quien atendiendo a planteamientos y gestiones de mi Gobierno, el 26 de noviembre de 1971, resolvió confirmar bienes comunales por 614,321 hectáreas al núcleo de compatriotas que habita el área de la selva lacandona (Velasco, 1972: 75).

El gobernador Velasco Suárez nunca se imaginó que esta decisión, considerada como un acto revolucionario, generaría uno de los mayores conflictos en la Selva Lacandona. Hoy viven en la Selva poco más de 406 mil habitantes, casi tres veces más que hace dos décadas. El desbordamiento de la población ha provocado que la Selva se convierta de zona de fuerte atracción a zona de expulsión de población, mientras que el tema agrario y territorial adquiere centralidad, al que se añade la conflictividad entre los grupos y organizaciones con banderas y colores de diverso tipo.

Segundo ciclo: Las migraciones interestatales

A partir de la segunda mitad de la década de 1950, Chiapas comienza a registrar un saldo neto migratorio negativo. En 1960, el número de chiapanecos fuera de la entidad era de casi $68 \mathrm{mil}$, con lo que el saldo migratorio ascendió, en ese año, a -18,951 (Jáuregui y Ávila, 2007). Hacia los setenta, la construcción del proyecto turístico de Cancún, así como el descubrimiento,

$16 \frac{\text { PRIMER SEMESTRE } 2014}{\text { MIGRACIÓN Y DESARROLLO NÚM. } 22}$ 
exploración y explotación de grandes yacimientos petrolíferos en el estado de Tabasco y norte de Chiapas, generan un mercado laboral, sobre todo en la industria de la construcción, que atrae a grandes contingentes de chiapanecos. En 1970 habían emigrado 90,578 chiapanecos, 70\% de estos concentrados en el Distrito Federal, Tabasco y Veracruz (Gobierno de Chiapas, 2006), con lo que el saldo neto migratorio fue de $-55,037$ personas.

En efecto, las migraciones interestatales se perfilan de manera más clara a partir de los años setenta, momento en el que ocurren varios acontecimientos. Por su impacto se pueden destacar tres:

1. Permanencia de una estructura agraria concentrada en grandes latifundios y neolatifundios, frente a una masa de campesinos sin tierra, situación que generó fuertes conflictos agrarios.

2. El descubrimiento de grandes yacimientos de petróleo y gas en el área norte de Chiapas y territorios de Tabasco.

3. La construcción del megaproyecto turístico de Cancún, en el estado de Quinta Roo.

Estos tres hechos generan una fuerza de atracción para miles de chiapanecos que se encontraban en el límite de la subsistencia. En una década y media — de 1970 a 1985—, los chiapanecos aprendieron a desplazarse lejos de sus tierras. Esta experiencia, junto con la formación de redes de migración centroamericana, aunada a la descomunal crisis de los principales productos del campo, el levantamiento armado del Ejercito Zapatista de Liberación Nacional (EZLN) y los desastres causados por los huracanes Mitch en 1998 y Stan en 2005, ${ }^{4}$ posibilitarán más adelante la migración internacional.

Frente a la relevancia que ha venido adquiriendo la migración internacional de chiapanecos, las migraciones interestatales han perdido visibilidad a pesar de su creciente importancia. En 1990, el número de chiapanecos

${ }^{4}$ Arévalo (2009), con base a información de la Comisión Económica para América Latina y el Caribe (CEPAL), estima que el número de damnificados directos por ambos fenómenos meteorológicos sumaron 282,578, concentrando 90\% en el caso de Stan; 315 muertos, la mayoría correspondieron a los efectos del Mitch, y los daños directos ascendieron a 1,439.7 millones de dólares. 
fuera del estado fue de 229,372, con un saldo neto migratorio de $-122,342$ (Jáuregui y Ávila, 2007). Luego del levantamiento zapatista, de la persistencia de la crisis de los precios agrícolas — principalmente el café_ y de los desastres causados por los fenómenos hidrometeorológicos antes referidos, la emigración se intensificó de tal forma que en 2000 el número de chiapanecos fuera de la entidad ascendió a 336,140, con un saldo migratorio de -213,689. De acuerdo con la información más reciente, en 2010 el número de chiapanecos fuera de la entidad ascendió a 491,919, con un saldo de $-351,784$ (INEGI, 2013). Se trata de una cifra histórica con lo que, Chiapas, junto con Guerrero y Tabasco, se perfiló como una entidad expulsora por excelencia de población.

Un hecho significativo es el crecimiento que en los últimos años registra la emigración de chiapanecos a las entidades fronterizas del norte del país. En 2000, si bien los migrantes chiapanecos se concentraron en el Estado de México, Distrito Federal, Quintana Roo y Campeche, Baja California fue el lugar de destino de 20,214 chiapanecos, y más de 30 mil se habían trasladado con fines laborales a Tamaulipas, Chihuahua, Jalisco, Nuevo León, Sonora y Sinaloa. La reestructuración económica generó una relocalización del capital, convirtiendo a esas entidades en zonas de mayor desarrollo, por sus ventajas en recursos productivos y mercados laborales: maquiladoras, agricultura de exportación y servicios. La migración femenina a esos estados es visible, como consecuencia de los procesos de deslocalización industrial del capitalismo global que se expresa en la creación de zonas francas, donde el trabajo asalariado femenino representa el factor distintivo del proletariado de estas industrias.

La migración interestatal a finales de los noventa mostró un nuevo rostro, marcado por una economía abierta que generó la proliferación de la industria maquiladora, el desarrollo de polos turísticos y el apoyo de sectores agrícolas dinámicos como la agroindustria tequilera en Los Altos de Jalisco que demandó cantidades importantes de fuerza de trabajo del sur del país, en especial de Chiapas. En este proceso es interesante ver que en el periodo 2005-2010 el principal destino de los migrantes chiapanecos fue Quintana Roo, lo cual se explica por la gran demanda de mano de obra en la industria de 
la construcción. En esta nueva dinámica, el Distrito Federal y el Estado de México dejaron de ser los centros de atracción de migrantes por excelencia perfilándose un patrón más diversificado, con miras hacia el norte. Sin embargo, la violencia que empezaron a experimentar ciertos lugares como Tijuana y después Ciudad Juárez desincentivó la migración hacia estos destinos.

Haciendo abstracción del Estado de México, que desde décadas atrás ha sido un lugar significativo para la migración de chiapanecos, en 2010 tres estados ocuparon un sitio destacado en la migración interestatal: Quintana Roo con 67,039; Tabasco, 60,336; Baja California, 52,119. Otros estados del norte como Baja California Sur, Chihuahua, Nuevo León y Sonora sumaron 36,245 chiapanecos; además figuran Nayarit y Jalisco que sumaron 22,509, la mayor parte localizados en este último. No obviamos, desde luego, la importancia que tuvieron los estados de Tabasco, Veracruz y Campeche, que juntos sumaron 123,743, casi la mitad se concentró Tabasco.

Quintana Roo es la entidad que resultó con el más alto porcentaje de población nacida en otros estados del país, con casi $53 \%$, al que le sigue Baja California con $41.2 \%$. Como hemos apuntado arriba, la cantidad de chiapanecos residentes en ambos estados es muy significativa, de hecho ocupan el primer y el cuarto lugar, respectivamente. Después de la crisis de 2009, registraron fuertes caídas en su crecimiento económico; sin embargo entre 2010 y 2011 ambos estados observan un significativo incremento en el PIB, por arriba del promedio nacional, que fue de 3.9\%: Baja California presentó una tasa de 6.5\% y Quintana Roo de 5.2\%, en tanto que Chiapas sólo registró un $3.4 \%$. La industria de la construcción, que fue afectada por la crisis, creció $4.6 \%$ a nivel nacional, en Baja California a 12.9\%, en Quintana Roo a $7.2 \%$ y en Chiapas a $-6.6 \%$.

Lo que importa subrayar es que durante el periodo 2000-2010 Chiapas se consolida como una de las entidades expulsoras de población, de hecho ya en 2005 se ubicó en el segundo lugar, después del Distrito Federal, con mayor saldo migratorio negativo, lo que evidentemente revela un problema de fondo, relacionado fundamentalmente con la precariedad de las condiciones económicas que obliga a la población a desplazarse a otras entidades que ofrecen mayores oportunidades. Como referimos al principio, Chiapas sigue 
ocupando el primer lugar en pobreza y en niveles de marginación, que aunado a la crisis del sector rural, constituyen elementos fundamentales que explican el éxodo al interior del país y hacia Estados Unidos. El sector informal ha sido durante la última década uno de los espacios de refugio de gran cantidad de gente del campo.

Tercer ciclo: las migraciones internacionales

¿Por qué es importante el estudio de la migración internacional en Chiapas? Ésta es una pregunta clave para entender lo que ha ocurrido después de turbulento periodo de 1994-2000 y el escenario en la década posterior. En 1994 ocurre el levantamiento armado del EzLN y junto con este proceso se desencadenan varios fenómenos: desplazamientos de población, toma de tierras, surgimiento de una gran cantidad de organizaciones de la sociedad civil, organizaciones de corte contrainsurgente y militarización. A partir de 2000, en medio de la alternancia política, tanto federal como estatal, se produce un incremento sustancial en los flujos migratorios que se refleja en el monto de las remesas. En este sentido, la migración de chiapanecos vendría a generar un proceso de distensión que se evidencia en una menor demanda agraria y de apoyos a la agricultura en fase de crisis profunda. Sin embargo, las remesas no han superado la pobreza estructural y tampoco la nueva pobreza; la crisis que comenzó a finales de 2007 en Estados Unidos afectó el envío de remesas y en Chiapas no se crearon condiciones para superar los rezagos sociales y económicos, de manera que hoy los sectores mayoritarios de la sociedad se encuentran sin salida.

La migración internacional en Chiapas aparece como por arte de magia a principios de los años de 1990. La noticia histórica de que Chiapas se estaba insertando al tren de la globalización por la vía de la exportación de mano de obra barata a Estados Unidos sorprendió a propios y a extraños, y llevó a preguntarse sobre las causas y consecuencias. Durand y Massey, al dilucidar las migraciones en el sureste, plantean que «más que explicar su origen migratorio

$20 \frac{\text { PRIMER SEMESTRE } 2014}{\text { MIGRACIÓN Y DESARROLLO NÚM. } 22}$ 
habría que explicar por qué esta región permaneció al margen y cuál podría ser su futuro inmediato» (2003: 89). En seguida expresan que:

En el caso de Chiapas, visto desde la óptica migratoria, resulta paradójico: la ausencia de migración puede explicar la guerra, y ésta puede explicar la migración, dicho de otro modo, la migración y las remesas parecen haber operado como un paliativo de los conflictos sociales en situaciones similares, como las ocurridas en Guerrero y Oaxaca. Por otra parte, la guerra de baja intensidad en el estado de Chiapas, que lleva más de un lustro, ha generado desplazamientos de población, y se sabe que este factor, al igual que en Centroamérica, puede convertirse en un elemento catalizador del flujo migratorio (Durand y Massey, 2003: 90).

La conflictividad desatada con la aparición pública del ezLn y el vínculo con la migración internacional, consideramos que es un factor, entre muchos otros que tienen mayor peso, toda vez que la migración internacional comenzó en regiones con menor presencia del ezLn, como La Sierra, Costa y Soconusco. Desde luego, se registraron desplazamientos, pero éstos fueron fundamentalmente de carácter interno, aunque después se aprecia una migración internacional de municipios que formaron parte del escenario de conflicto, tal es el caso de Las Margaritas. Es cierto que más adelante la migración y las remesas contribuyeron a bajar la presión social, que fue muy evidente en el problema agrario donde a partir de 2000 fue disminuyendo de intensidad y que prácticamente se prolongó hasta la mitad del sexenio de Juan Sabines. Es decir, durante el periodo 2000-2009, las invasiones de tierras fueron muy esporádicas.

En consecuencia, el fenómeno migratorio reviste un carácter complejo que obliga a hurgar más allá de lo aparente, con una visión articulada que incluye las condiciones materiales, sociales, políticas, así como las aspiraciones de los jóvenes que reclaman una vida más allá de las fronteras del medio rural, que ya no genera empleos y tampoco garantiza la autosuficiencia alimentaria, menos aún las condiciones de reproducción social y simbólica. La emergencia del fenómeno migratorio internacional en Chiapas coincide 
con la convergencia de varios acontecimientos en un periodo de aplicación de las políticas neoliberales que fueron devastadoras en las condiciones económicas y sociales de Chiapas donde el Estado era prácticamente el único inversionista.

La incorporación de los chiapanecos al circuito de la migración internacional es relativamente reciente, no más allá de dos décadas. Las primeras experiencias se registran en 1989, en la región Sierra, año en que los cafeticultores vivieron una de las peores crisis de los precios internacionales del café.

El fenómeno migratorio no fue objeto de atención y de preocupación del aparato gubernamental que tenía puesta la mira en otros fenómenos que ocuparon la centralidad en el quehacer político y en el análisis de la conflictividad social. Por ejemplo, el levantamiento zapatista opacó otros fenómenos, incluyendo la migración internacional. Incluso, aún después de la turbulencia zapatista, a finales de los noventa, había cierta reticencia en reconocer la importancia de este fenómeno de parte de las autoridades gubernamentales y el medio académico.

Una de las razones que explican esta actitud deriva de la inexistencia de una tradición migratoria internacional y de las propias fuentes estadísticas. En efecto, aún las estadísticas más recientes no revelan la importancia del fenómeno migratorio. El índice de intensidad migratoria, según datos del Consejo Nacional de Población (Conapo, 2012), para 2010 fue de -0.9913, considerado muy bajo. Para el mismo año, sólo seis municipios registraron intensidad media, ${ }^{5} 30$ de baja intensidad y 80 muy baja.

Lo inédito de la migración internacional de entidades federativas como Veracruz y Chiapas es su extraordinario crecimiento en un periodo relativamente corto. En las entrevistas que hemos realizado, hay un dato casi generalizado: en su mayoría inician la experiencia migratoria internacional entre 2000 y 2007. También es extraordinario su dinamismo, al grado de ocupar hoy la centralidad de la vida económica de muchas localidades y municipios. Estos elementos, además de operarse en un contexto de globalización neoliberal,

${ }^{5}$ Amatenango de la Frontera, Bella Vista, La Margaritas, Unión Juárez y La Trinitaria.

$22 \frac{\text { PRIMER SEMESTRE } 2014}{\text { MIGRACIÓN Y DESARROLLO NÚM. } 22}$ 
marcan sin duda diferencias sustantivas con respecto a otras entidades con tradición migratoria internacional.

Un hecho significativo es el crecimiento que en los últimos años registra la emigración de chiapanecos a las entidades fronterizas del norte del país. En efecto, en un breve tiempo las «agencias de viajes» que hacen el recorrido hasta Tijuana, han proliferado en las localidades como Las Margaritas, Frontera Comalapa y Motozintla, municipios de intersección entre dos regiones de alta migración, como las regiones Sierra y Fronteriza. Tan sólo en Frontera Comalapa identificamos en 2005 cerca de 15 «agencias de viajes», aunque sólo salían tres autobuses a la semana por ser "temporada mala», en contraste con los meses de principio de año cuando salen de seis a siete autobuses a la semana. El itinerario registrado por estas empresas es similar: Ciudad Juárez, Agua Prieta, Altar Sonora, Mexicali y Tijuana.

Estas agencias también son muy visibles en Tuxtla Gutiérrez, capital del estado de Chiapas, Tapachula, San Cristóbal de Las Casas, Altamirano, Villa Corzo y Villa Flores. El espejismo del dólar ha penetrado hasta en las comunidades más apartadas del estado chiapaneco, provocando un efecto de arrastre sólo mitigado, temporalmente, por la crisis de la economía estadounidense y el reforzamiento de las medidas de control al tránsito. A este respecto, un reportaje de Bellinghausen refiere:

En los pueblos indígenas de la selva y la frontera se ha vuelto una verdadera «moda» unirse a una cuadrilla de pollos. En las afueras de Ocosingo, Comitán, Las Margaritas o La Trinitaria abundan oficinas de tijuaneros que ofrecen viajes a Tijuana y Altar «todos los miércoles, a precios económicos». Anuncian escalas en Tuxtla Gutiérrez, Oaxaca, Querétaro, San Luis Potosí, Zacatecas, Agua Prieta, Nogales. Y «absoluta seriedad» (Bellinghausen, 2005).

En este contexto resulta pertinente formular las siguientes preguntas: ¿̇cuáles son las causas de la migración internacional?, żquiénes emigran?, żqué regiones o municipios participan de la emigración internacional? ìhacia qué regiones de Estados Unidos emigran los chiapanecos? 
En la explicación de la emergencia de la migración internacional en Chiapas aparecen dos factores: la crisis del café y la profundización de la pobreza rural, a instancias de un conjunto de factores. Los dos están articulados y se explican por una crisis de mayor envergadura en el sector agropecuario, provocada en gran parte por las medidas que acompañaron a la apertura comercial, como la disminución del gasto público en materia de subsidios, apoyos a la comercialización, créditos para la producción y creación de infraestructura que generó una brutal caída de la producción, que se reflejó en un decremento del PIB sectorial durante tres años consecutivos. A esto se suma el conflicto ocurrido a partir de la aparición del EZLN. Un tercer factor agregado fueron los efectos de dos huracanes que provocaron daños considerables a las economías campesinas de las regiones Costa y Sierra: el Mitch en 1998 y el Stan en 2005.

Chiapas ocupa el primer lugar en la producción nacional de café, ${ }^{6}$ un producto que demanda grandes cantidades de mano de obra y que se ha visto sometido a dos severas crisis por la baja en el precio internacional, y tal parece que ahora estamos en la antesala de una tercera. ${ }^{7}$ La primera a finales de los años ochenta, que se prolonga hasta 1995 y la segunda ocurre en 1997, luego de dos años de una lenta recuperación. En 2005 de nuevo comienzan a mejorar los precios, pero entonces muchos campesinos habían abandonado

${ }^{6}$ De acuerdo con el Servicio de Información Agropecuaria y Pesquera, de la Secretaría de Agricultura, Ganadería, Desarrollo Rural, Pesca y Alimentación (2010), el número de productores de café registrados es de 183,761 y la superficie cultivada asciende a 241,876 hectáreas, distribuidas en 198,320 predios.

7 En efecto, de acuerdo con la Organización Internacional del Café (OIC, 2013), el precio compuesto en los mercados de futuro ha venido cayendo desde marzo de 2012 cuando alcanzó 167.77 centavos de dólar la libra a marzo de 2013 que llegó a 131.38, lo que representa una caída de $21.7 \%$. De manera paralela, la cafeticultura enfrenta un serio problema de roya que se traduce en una baja producción y pérdida de empleos. En el caso de Centroamérica la superficie de café afectada por esta plaga asciende a 482,146 hectáreas y la pérdida de 351,000 empleos. En Chiapas se estima que 45 mil hectáreas —alrededor de 18\% de la superficie cultivada - están afectadas por la roya. El cambio climático está influyendo en los impactos: "hace tres décadas la roya impactaba hasta 800 metros sobre el nivel del mar y ahora llega a cultivos ubicados hasta mil 460 metros sobre el nivel del mar» (Comcafe, 2013).

$24 \frac{\text { PRIMER SEMESTRE } 2014}{\text { MIGRACIÓN Y DESARROLLO NÚM. } 22}$ 
el campo a fin de pagar deudas y tener un mínimo de ingresos para asegurar la reproducción de la familia.

La migración internacional en Chiapas, el estado que ocupa el primer lugar nacional en marginación y pobreza, está relativizando la idea, por cierto muy difundida entre los estudiosos del fenómeno migratorio, según la cual no es la población más pobre la que emigra, porque esto supone contar con recursos financieros (Castles, 2000; Portes y Rambaut, 2010). Sin embargo, no debemos olvidar que el capital social, quizá tan importante como el capital financiero, juega un papel definitorio en el proceso de migración.

¿Quiénes emigran? Esta interrogante resulta problemática. Para las autoridades del estado de Chiapas, y para la mayoría de los actores políticos, era muy recurrente minimizar el fenómeno migratorio internacional; cuando este fenómeno se visibilizó, se empezó a argumentar que quienes emigraban no eran los «pobres», sino quienes tenían un poder adquisitivo suficiente para costear una travesía relativamente cara. Estas afirmaciones no corresponden del todo con las informaciones recuperadas en el trabajo de campo y con las declaraciones de muchas organizaciones sociales y actores políticos locales.

El grupo de las personas que emigran a Estados Unidos se ha ampliado en la medida en que la crisis se profundiza. Emigran los que tienen recursos y buscan mejorar sus condiciones de vida; también lo hacen quienes logran acceder a un préstamo a cambio de dejar las escrituras de una propiedad y pagar altos intereses; emigran quienes consiguen el dinero para el viaje a partir de los apoyos de la familia o un préstamo bajo la promesa de su pago por parte de la familia. Están emigrando los pobres y ello fue posible en sus inicios por las ofertas de trabajo que las empresas camioneras hacen en municipios claves. Están emigrando los más pobres, los indígenas que no alcanzan a reproducirse con sus minúsculas parcerlas de menos de una hectárea. ${ }^{8}$

Algunos datos que pueden ayudar a formular hipótesis sobre quiénes emigran son las matrículas consulares que se han venido incrementado en la última década. De acuerdo con la información de la Secretaría de Relaciones

${ }^{8}$ Véase la bien documentada tesis doctoral de Coporo (2013) sobre el caso Chamula. 
Exteriores (SRE), en 2001 se expidieron 4,092 matrículas consulares, situando a los chiapanecos como el grupo poblacional con el mayor número en ese año. En 2003 la expedición de matrículas alcanzó la cifra de 9,852. La SRE reporta que los consulados con el mayor número de expediciones a chiapanecos fueron de los estados de California, Florida y Texas. En 2011, el número de matrículas se había incrementado en $31.5 \%$, con relación a 2003 y en $217 \%$, lo que refleja la importancia que va adquiriendo la migración en Chiapas.

En 2011 se expidieron 12,960 matrículas en 45 consulados de México en Estados Unidos; sin embargo, sólo tres de éstos concentraron 4,583 matrículas, es decir, $35.4 \%$ del total, dato que puede ser indicativo del lugar donde se concentran los chiapanecos, por orden de importancia, son los siguientes: Orlando con 1,592; Atlanta con 1,571; y Raleigh, con 1,430. En Atlanta prácticamente está representado 75\% de los municipios de Chiapas, destacando por el número de matrículas Tapachula, Frontera Comalapa, La Trinitaria y Motozintla; le sigue otro grupo compuesto por Chicomuselo, Huehuetán, Tuxtla Chico, Silpepec, Cacahuatán, Mapastepec, Villa Comaltitlán y Chamula. En el consulado de Orlando el mayor número de matrículas fue expedido para originarios de Chamula, San Cristóbal de Las Casas, Tapachula y Frontera Comalapa; y en Raleigh destacan los de Tapachula, Motozintla y Frontera Comalapa. Como se puede observar, Tapachula y Frontera Comalapa se repiten en los tres consulados, lo que da una idea de la importancia de la migración internacional en estos dos municipios, que por cierto no aparecen en la lista del Conapo de municipios con índice de intensidad migratoria medio.

Por estados, las matrículas se concentraron en orden de importancia en California, Florida y Texas, que sumaron 5,943, equivalente a 45.8\%; le sigue Georgia y las Carolinas, que juntos reunieron 2,328 matrículas, es decir, $18 \%$. Además, de acuerdo con la información del gobierno de Chiapas, en 2010 se identificaron 11 clubes de oriundos y en 2012 sumaban 18 organizaciones de chiapanecos en Estados Unidos. Entre 2011 y 2012 se formaron las siguientes organizaciones: Federación de Chiapanecos en el Exterior; Federación de Chiapanecos en el Sur de California; Federación de Chiapanecos en Nueva York, y la Confederación de Federaciones de Chiapanecos en Estados 
Unidos (Gobierno de Chiapas, 2013). De acuerdo con la información de la SRE, sólo en 2011 se registraron cinco asociaciones: Sembrando por Chiapas, con residencia en Dallas; Mi Tierra Arriaga, en Los Ángeles; Arriaga, en Filmore, California; Somos Muchos Chiapanecos en el Extranjero, en Phoenix, Arizona; y Lacandones, en Salt Lake City, Utah.

Otra variable interesante es el nivel educativo de los chiapanecos que obtuvieron matrículas consulares en 2011: de las 12,960 matrículas, 347 $(2.7 \%)$ correspondieron a personas sin estudios; 1,858 (14.3\%) con primaria incompleta; $2,813(21.7 \%)$ con certificado de primaria; 4,316 con certificado de secundaria; y 2,083 con certificado de preparatoria, el resto se distribuye entre capacitación técnica y preparatoria sin certificado. Es decir, casi 39\% de los migrantes con matrículas consulares se situaron en un rango de calificación que va de no tener estudios hasta la primaria con certificado. Suponemos que estas calificaciones corresponden, en su mayoría, a personas que provienen del campo, sin oportunidades para estudiar más allá de la primaria.

En correspondencia con los datos anteriores, el perfil ocupacional de los migrantes se distribuye como sigue: 6,794 (52.42\%) corresponde a empleado; 2,141 (16.52\%) a ocupación en el hogar; 2,276 (17.56\%) a las categorías de obrero, campesino y jardinero; y 666 (5.15\%) a cocinero, pintor y albañil. Desafortunadamente, no se desglosa la categoría de empleado para tener mayor precisión sobre las características del empleo, pero suponemos que se trata empleos precarios dado el nivel de educación predominante.

Es evidente que estas cifras, aún cuando son relevantes, no reflejan la magnitud de la emigración de chiapanecos a Estados Unidos, pues sólo un sector minoritario de migrantes tramita la matrícula consular que otorga el gobierno mexicano, y sin embargo abona a la hipótesis sobre la creciente importancia de la migración de chiapanecos a Estados Unidos, que se ha manejado muy discretamente, quizá porque refleja el fracaso de la política social, sobre todo de combate a la pobreza.

Sin lugar a duda, una fuente que refleja con mayor realismo, aun con todas las limitaciones, es la Encuesta sobre Migración en la Frontera Norte (EMIF), que registra los movimientos de la población que provienen del sur hacia el norte, así como los que proceden del norte con destino a sus entidades 
federativas de residencia. En este caso tomamos la información de lugar de destino y entidad federativa, que refleja bastante bien la intensidad del flujo migratorio y el destino del mismo.

Como se puede apreciar en el cuadro 3, se registra un punto de inflexión a partir de 2002, tanto en lo que se refiere a la intensidad del flujo migratorio como en el destino de la migración de los chiapanecos. Hasta ese año, la mayor cantidad tenía como destino a la frontera norte y una proporción bastante menor a Estados Unidos. Sin embargo, como se observa en los años siguiente, se registra un cambio radical en la orientación. Así, mientras que en 1999 poco más de 87\% de los migrantes chiapanecos se dirigía a la frontera norte, en 2002 apenas llegó a 37\%. En adelante, los flujos se orientarán preferentemente a Estados Unidos, durante los últimos cuatro años de registros de la Encuesta (2005-2008), se observa un predominio absoluto de la migración hacia Estados Unidos alcanzando las mayores valores en 2007 y 2008 con $86.4 \%$ y $83.9 \%$, respectivamente.

\section{CUADRO 3}

Migrantes con residencia en Chiapas con destino a la frontera norte y a Estados Unidos

\begin{tabular}{lcrr}
\hline AÑO & A LA FRONTERA NORTE & A ESTADOS UNIDOS & TOTAL \\
\hline 1995 & 3,502 & 2,434 & 5,936 \\
1999 & 22,942 & 3,391 & 26,333 \\
2000 & 42,960 & 3,446 & 46,407 \\
2001 & 30,022 & 4,472 & 34,494 \\
2002 & 21,458 & 36,307 & 57,765 \\
2003 & 15,922 & 53,828 & 69,750 \\
2004 & 25,928 & 33,786 & 59,714 \\
2005 & 21,548 & 60,427 & 81,975 \\
2006 & 21,724 & 118,510 & 140,234 \\
2007 & 22,598 & 143,939 & 166,537 \\
2008 & 26,085 & 136,388 & 162,472 \\
\hline
\end{tabular}

Fuente: STPS, Conapo, INM, SRE y Colef.

Otro dato interesante de la EmIF es el registro del crecimiento del flujo migratorio de chiapanecos, tanto hacia la frontera norte como a Estados

$28 \frac{\text { PRIMER SEMESTRE } 2014}{\text { MIGRACIÓN Y DESARROLLO NÚM. } 22}$ 
Unidos. Así, en el periodo 2000-2007, la Encuesta muestra un crecimiento de $259 \%$, en 2008 hay una leve caída de $2.4 \%$, que puede explicarse por la crisis económica y laboral en Estados Unidos, pero que no demerita en absoluto la tendencia que viene a confirmar la importancia de la migración internacional en Chiapas y que, de alguna manera, cuestiona las cifras censales.

Otro dato relevante de la EMIF se refiere a las deportaciones de la Patrulla Fronteriza, mismas que van teniendo un peso mayor en suma de las devoluciones totales que va de un $0.7 \%$ a poco más del $10 \%$ registrado en 2005 . Estos datos reflejan también la condición irregular de los migrantes chiapanecos, sus condiciones de vulnerabilidad, así como su reciente ingreso a las corrientes de la migración internacional.

CUADRO 4

Migrantes chiapanecos devueltos por la Patrulla Fronteriza

\begin{tabular}{cccc}
\hline AÑO & MIGRANTES CHIAPANECOS & TOTAL NACIONAL & PORCIENTO RESPECTO AL TOTAL \\
\hline 1995 & 4,731 & 636,878 & 0.7 \\
1999 & 15,182 & 619,556 & 2.4 \\
2000 & 32,269 & 772,294 & 4.2 \\
2001 & 17,448 & 590,732 & 2.9 \\
2002 & 39,258 & 536,334 & 7.3 \\
2003 & 36,835 & 426,027 & 8.6 \\
2004 & 26,389 & 402,449 & 5.5 \\
2005 & 48,745 & 479,368 & 10.2 \\
2006 & 31,911 & 442,774 & 7.2 \\
2007 & 51,744 & 536,255 & 9.6 \\
2008 & 45,711 & 513,241 & 8.9 \\
\hline
\end{tabular}

Fuente: STPS, Conapo, INM, SRE y Colef.

En el cuadro 4 resulta evidente el salto cuantitativo en el número de chiapanecos deportados por la Patrulla Fronteriza entre 1995 y 2008, es decir, entre el momento en que la migración hacia Estados Unidos comienza y en los años donde ya se ha establecido como un fenómeno sostenido. Se trata de un incremento de $866 \%$, lo que revela el crecimiento del flujo migratorio, toda vez que existe una correlación positiva entre el aumento de la migración 
y el aumento de detenciones y devoluciones. En 2005, Chiapas ocupa el primer lugar nacional en devoluciones; en 2006 pasa al tercer sitio, seguido de Michoacán y Veracruz; en 2007 se coloca en segundo lugar, después de Oaxaca; y en 2008 de nuevo recupera el primer lugar.

\section{EL ESPEJISMO de LAS REMESAS, LA CRISIS Y EL ESCENARIO TENDENCIAL}

En contraste con la escasez de datos sobre el número de migrantes chiapanecos en Estados Unidos, la fuente más usada sobre remesas es el Banco de México (Banxico), que sistemáticamente publica cada trimestre. Algunos estudiosos del fenómeno migratorio se han escandalizado con las cifras sobre remesas presentadas por el Banxico, por considerarlas muy altas dada la baja intensidad migratoria de la entidad. En consecuencia, suponen un problema metodológico en la contabilidad o en todo caso se plantea la hipótesis en torno a una relación con el narcotráfico (véase Tuirán, Santibáñez y Corona, 2006). ${ }^{9}$ ¿ Qué intereses tendrían los economistas del Banxico para alterar las cifras y hacer aparecer a México y, en particular, algunos estados como Chiapas, con cifras que aparentemente resultan incongruentes con el flujo de migrantes? Más allá de este debate, que por cierto no tuvo ninguna incidencia en la contabilidad del Banxico, podemos decir que, aun si se tratara de cifras más conservadoras, estarían reflejando la importancia estratégica que tiene la migración para una buena cantidad de familias campesinas chiapanecas que se encontraban en los límites de la subsistencia.

${ }^{9} \mathrm{Al}$ respecto, Canales dedica un artículo para dilucidar el problema planteado por los autores citados y compara varias fuentes para llegar a la siguiente conclusión: «[...] se puede afirmar que el problema probablemente se presente en la nueva metodología de estimación del Banxico. Por lo pronto, con la metodología usada con anterioridad a 2001, todas estas comparaciones arrojan similares consistencias entre lo reportado por Banxico y el Buró de Análisis Económico del Departamento de Comercio de Estados Unidos» (Canales, 2008: 34). Delgado, Márquez y Rodríguez (2009) hacen un análisis en el que sitúan el debate de las remesas más allá de las cifras del Banxico, demostrando que las remesas que envían los mexicanos sólo constituye una mínima parte de lo que generan los migrantes con su trabajo en Estados Unidos.

$30 \frac{\text { PRIMER SEMESTRE } 2014}{\text { MIGRACIÓN Y DESARROLLO NÚM. } 22}$ 
Una somera revisión de la cifras presentadas por el Banxico durante el periodo 2003-2011 sorprende por el rápido crecimiento hasta 2008, en adelante bajarán por el efecto combinado de la crisis económica en Estados Unidos que afectó áreas sensibles como la industria de la construcción donde se insertan los migrantes y el endurecimiento de las medidas de control en la frontera.

\section{CUADRO 5}

Producto Interno Bruto del sector primario y remesas familiares

(Millones de pesos corrientes)

\begin{tabular}{cccc}
\hline AÑO & PIB SECTOR PRIMARIO & REMESAS & REMESAS/PIB SECTOR PRIMARIO (\%) \\
\hline 2003 & $13,636.7$ & $4,889.3$ & 35.85 \\
2004 & $14,313.6$ & $6,590.7$ & 46.04 \\
2005 & $14,053.2$ & $8,196.9$ & 58.32 \\
2006 & $16,210.6$ & $10,231.6$ & 63.11 \\
2007 & $17,259.8$ & $10,043.9$ & 58.19 \\
2008 & $18,613.0$ & $11,171.9$ & 60.02 \\
2009 & $18,592.6$ & $7,952.7$ & 42.77 \\
2010 & $20,530.2$ & $7,112.0$ & 32.64 \\
2011 & $24,221.0$ & $8,314.5$ & 34.32 \\
\hline
\end{tabular}

Nota: los dólares fueron convertidos a pesos, de acuerdo a la paridad de cada año.10 Fuente: INEGI (2013) y Banxico (2013).

Lo interesante de estos datos — con todas las reservas que puedan tener- es que frente a la crisis rural, las remesas están supliendo los ingresos antes generados por la agricultura y la ganadería ¿̇Qué significa este hecho? Se pueden hacer varias lecturas en distintas direcciones, una de ellas es que si bien es un hecho que las remesas están ocupando la centralidad que tenía la parcela, representan una fuente menos estable, con un costo social muy alto: desde 2008 las remesas han venido bajando, y si bien en 2011 aparece un incremento en pesos es porque el tipo de cambio presentó una variación.

Estamos frente a la probabilidad de tener una dependencia estructural de las remesas que puede significar un riesgo frente a la posibilidad de ${ }^{10}$ Los datos de paridad peso-dólar fueron tomados de Aguirre (2013). 
aprobación de la reforma migratoria, que dejaría sin posibilidades de quedarse en Estados Unidos a la gran mayoría de los chiapanecos por no cumplir con los requisitos básicos, que entre otras cosas implica registrarse, someterse a revisión de antecedentes criminales, pagar multa, impuestos, aprender inglés e historia cívica, además de que se incrementaría la seguridad fronteriza y se sancionaría a las empresas que contraten trabajadores indocumentados.

Hasta ahora no hay evidencias de que las remesas hayan generado procesos de desarrollo local, incluso los indicadores oficiales presentan un incremento en el número total de pobres, a pesar de una leve reducción en el número de pobres extremos. No hay visos de una reactivación de la producción agropecuaria y una recuperación de la economía campesina que ha sido prácticamente desmantelada con las políticas neoliberales que reclasificaron a los productores y enviaron a los programas asistenciales a la inmensa mayoría de campesinos.

El agro chiapaneco vive un proceso de des-acumulación, las cifras más recientes revelan que la contribución del sector primario al PiB estatal es de 9\% (INEGI, 2013). Incluso trabajadores centroamericanos que tradicionalmente llegan a Chiapas para realizar actividades de la agricultura de plantaciones, se han venido desplazando al sector de servicios, y en otros casos a la construcción, en el Caribe mexicano. A este respecto, es importante anotar que entre las respuestas recurrentes sobre el uso de las remesas, no se registra la inversión en la parcela.

El mayor mérito de las remesas ha sido atenuar la conflictividad social, expresada en otros momentos en invasiones de tierras y enfrentamientos entre comunidades. Hoy, sin embargo, comienzan a verse signos de una reactivación de las tensiones que se manifiesta en conflictos por límites territoriales en varias comunidades, en linchamientos, en la disputa por el control de lugares de alto valor escénico como es el caso de San Marcos Avilés, municipio de Chilón, en donde en 2010 fueron desplazados 170 bases de apoyo zapatistas. En este mismo municipio, en los primeros meses de 2013 han ocurrido conflictos en el ejido San Sebastián Bachajón que alcanzó el nivel más alto de tensión con la muerte de Juan Vázquez, dirigente pro zapatista. En 
el ejido Tila, un grupo de campesinos reclama la restitución de 130 hectáreas y en los municipios de Motozintla y El Porvenir, en la Sierra chiapaneca, las comunidades se organizan en contra de la minería y de las altas tarifas de la luz eléctrica. En otros puntos de la geografía también se registran conflictos por la tierra, es el caso de los municipios de Rayón y Chapultenango donde se disputan poco más de 2,000 hectáreas.

El escenario para Chiapas y para los migrantes no es muy halagüeño, la reforma migratoria en ciernes no será una buena noticia para los chiapanecos. Su trayectoria migratoria en el más adverso de los escenarios no permite augurar un futuro promisorio, porque además la situación de las finanzas públicas atraviesan por una de las peores crisis. Desde el comienzo, el gobierno de Manuel Velasco está aplicando una política de austeridad que mantiene parada la industria de la construcción, los recursos para apoyos a la producción no fluyen y tampoco hay un proyecto para reactivar la economía e impulsar el desarrollo.

\section{RefLeXión finAL}

La migración internacional en Chiapas sólo es posible entenderla como parte de un largo ciclo, de una cambiante realidad donde grandes grupos de población del campo, y en menor medida de la ciudad, van sufriendo los efectos de los modelos de desarrollo ensayados en el país. Las luchas agrarias y la capacidad de reproducción de la unidad doméstica familiar hasta mediados de los años ochenta fueron los grandes contenedores de la migración. Otro contenedor fue el mercado laboral que representó para los chiapanecos la industria petrolera en Tabasco y del turismo en Cancún. El Estado ejercía un papel importante en la reproducción de la familia campesina, a través de diversos mecanismos, como los subsidios indirectos a la producción, la creación de infraestructura y apoyos a la comercialización de productos tan importantes en el ingreso familiar como el maíz y el café. 
La pobreza estructural y la generada por las políticas neoliberales de los últimos años han producido niveles de deterioro sin precedentes en las unidades familiares, de manera que este factor aunado al ambiente de tensión sociopolítica, han generado el reciente proceso de migración de chiapanecos a Estados Unidos. Los desajustes en la unidad doméstica campesina, provocados por el cambio de modelo, han hecho que los programas asistenciales y de apoyo a la producción resulten insuficientes para restaurar el precario equilibrio que tenía y, por lo mismo, se ha incorporado la práctica migratoria como componente central de su reproducción. Esto ha sido constatado en las estadísticas más recientes de Consejo Nacional de Evaluación de la Política de Desarrollo Social (CONEVAL) que muestran un Chiapas inmerso en la pobreza. Por ello, no es casual que la Cruzada Nacional contra el Hambre del actual gobierno federal se haya anunciado en Las Margaritas por el presidente de la República y su gabinete, y que también se haya puesto en marcha en la localidad de Navenchauc, del municipio de Zinacantán.

Chiapas es una de las entidades más olvidadas de México, donde sus indicadores sociales se equiparan a los países más pobres de África, como Sierra Leona y Malawi. Los Altos de Chiapas y la Sierra son regiones paradigmáticas en marginación y pobreza, ahora vinculadas a las migraciones internacionales. Este puede ser un buen indicador para repensar las migraciones internacionales y preguntarse si en verdad los más pobres y marginados de este país no migran.

A pesar de que la migración de chiapanecos a Estados Unidos y a zona de Playa del Carmen se convirtió en un factor decisivo de distensión en el campo chiapaneco, la demanda agraria y los conflictos por la disputa de tierras ocupadas durante los años del movimiento zapatista, así como de los viejos problemas por límites de tierra entre comunidades indígenas, mantienen un perfil que por ahora es bajo, pero que puede subir de intensidad en los próximos años frente a la profundización del modelo económico neoliberal, la crisis de la industria de la construcción en Estados Unidos, así como las medidas de contención del gobierno estadounidense, incluyendo las aplicación de medidas antiinmigrantes en varios estados de ese país.

$34 \frac{\text { PRIMER SEMESTRE } 2014}{\text { MIGRACIÓN Y DESARROLLO NÚM. } 22}$ 


\section{REFERENCIAS}

Aguirre, Manuel (2013), «Devaluación-inflación México-USA», en: http://www. mexicomaxico.org/Voto/SobreVal02.htm (consultado el 15 de enero de 2013).

AréVAlo, Jorge (2009), "Crisis económica, mercados de trabajo y emigración de chiapanecos a Estados Unidos», en Jesús Arroyo y Salvador Berumen (coords.), Migración a Estados Unidos. Remesas, autoempleo e informalidad, México, Universidad de Guadalajara-Instituto Nacional de Migración.

Banco De MéXico (2013), «Balanza de Pagos. Remesas familiares por entidad federativa», en: http://www.banxico.org.mx/SieInternet/consultarDirectorioInternetAction. .do? accion $=$ consultarDirectorioCuadros\&sector $=1 \&$ sectorD escripcion $=$ Balanza (consultado el 20 de marzo de 2013).

BÁez, Mariano (1985), «Soconusco: región, plantaciones y soberanía», en Andrés Fábregas et al., La formación histórica de la frontera sur, Cuadernos de la Casa Chata núm. 24, Ciesas, México.

Bellinghausen, Hermann (24 de enero de 2005), «La emigración de Chiapas a eU arrasa comunidades e individuos», La Jornada, en: http://www.jornada.unam. mx/2005/01/25/017n1pol.php (consultado el 12 de octubre de 2012).

Canales, Alejandro (2008), «Las cifras sobre remesas en México. ¿̇Son creíbles?», Migraciones Internacionales, vol. 4, núm. 4.

CASTIES, Stephen, (2000), «Migración internacional a comienzos del siglo xxI: tendencias y problemas mundiales», Revista internacional de ciencias sociales, núm. 165.

CIES (Centro de Investigaciones Ecológicas del Sureste) (1977), Reporte Final de Actividades del Fideicomiso de la Selva Lacandona, San Cristóbal de Las Casas, Chiapas.

Comcafe (Comisión para el Desarrollo y Fomento del Café) (2013), «Conferencia de prensa de Jorge Valdemar Utrilla en torno al problema de la roya en Chiapas», en: http://www.anacafemexico.com/concrete/.

coneval (Consejo Nacional de Evaluación de la Política de Desarrollo Social) (2008), "Mapas de pobreza por ingresos», en: www.sedesol.gob. (2010), "Análisis y Medición de la pobreza», en: http://www.coneval.gob. mx/Medicion/Paginas/Medici\%C3\%B3n/Anexo-Estadistico-Pobreza-2010. aspx (consultado el 10 de febrero de 2012).

Coporo, Gonzalo (2013), Migración, pobreza y desarrollo: estudios de caso en dos comunidades del municipio de Chamula, en Los Altos de Chiapas, Tesis doctoral, Centro 
de Estudios Superiores de México y Centroamérica-UNICACH, San Cristóbal de Las Casas, Chiapas.

Durand, Jorge y Douglas Massey (2003), Clandestinos. Migración México-Estados Unidos en los albores del siglo XXI, México, Universidad Autónoma de ZacatecasMiguel Ángel Porrúa.

Fernández, Luis y María Tarrío (1983), Ganadería y estructura Agraria en Chiapas, México, Universidad Autónoma Metropolitana-Xochimilco.

Fernández, Ortiz, Luis M. y Robert Wasserstrom (1977), "Los municipios alteños de Chiapas (México) y sus relaciones con la economía regional: dos estudios de caso", Estudios Sociales Centroamericanos, año vi, núm. 17.

Gobierno de Chiapas (2006), La otra frontera. Política migratoria en Chiapas, Tuxtla Gutiérrez, Coordinación de Relaciones Internacionales.

(2013), "Chiapas en el exterior», en: http://www.spdfs.chiapas.gob.mx/ acercate/clubes.html (consultado el 12 de marzo de 2013).

INEGI (Instituto Nacional de Estadística, Geografía e Informática) (2008), II Conteo de Población y Vivienda, Aguascalientes, INEGI.

(2013), «Banco de Información Económica. Cuentas Nacionales, Producto Interno Bruto Por entidad Federativa», en: http://www.inegi.org.mx/sistemas/bie/ (consultado el 13 de marzo de 2013).

(2013), «Migración interna. Saldo neto migratorio por entidad federativa», en: $w w w$.inegi.org. $\mathrm{mx} /$ sistemas/sisept/default.aspx? $\mathrm{t}=\mathrm{mdemo137 \& s}$ (consultado el 13 de enero de 2013).

JÁuregui, José Alfredo y María de Jesús Ávila (2007), «Estados Unidos, lugar de destino para los migrantes chiapanecos», Migraciones Internacionales, vol. 4, núm. 1. MARTínez, Germán (1994), Plantaciones, trabajo guatemalteco y política migratoria en la Frontera Sur de México, Tuxtla Gutiérrez, Gobierno del Estado de Chiapas-Consejo Estatal de Fomento a la Investigación y Difusión de la Cultura-Dif-ChiapasInstituto Chiapaneco de Cultura.

Mauricio, Juan Manuel, Rubén Valladares y Héctor García (1984), Lacandona. Una incorporación anárquica al desarrollo nacional, San Cristóbal de Las Casas, Centro de Investigaciones Ecológicas del Sureste.

Morales, Eduardo et al. (1978), Los Altos de Chiapas. Una zona de reserva de mano de obra, México, Centro Nacional de Productividad de México. 
Organización Integral del Trabajo (oit) (2003), "El programa de Trabajo Decente», en: http://www.ilo.org/global/about-the-ilo/decent-work-agenda/lang-es/index.htm (consultado el 25 de marzo de 2013).

OIC (Organización Internacional del Café) (2013), «Informe mensual sobre el mercado del café, marzo», en: http://www.ico.org/documents/cy2012-13/cmr0313-c.pdf.

Portes, Alejandro y Rubén Rambaut (2010), América Inmigrante, España, Anthropos. STPS (Secretaría del Trabajo y Previsión Social) (2012), Encuesta Nacional de Ocupación y Empleo 2012, en: http://www.stps.gob.mx/bp/secciones/conoce/areas_ atencion/areas_atencion/web/menu_infsector.html.

-Conapo-INM-SRE-El Colef, Encuesta sobre Migración en la Frontera Norte de México (emif), Secretaría del Trabajo y Previsión Social. Estadísticas de productividad laboral, en: http://www.stps.gob.mx/bp/secciones/conoce/areas_ atencion/areas_atencion/web/menu_infsector.html.

Turrán, Rodolfo, Jorge Santibáñez y Rodolfo Corona (2006), «El monto de las remesas familiares en México żMito o realidad», Papeles de Población, núm. 50.

Velasco, Manuel (1972), Chiapas. Segundo Informe de Gobierno, Tuxtla Gutiérrez, Gobierno del Estado de Chiapas.

Viqueira, Juan Pedro (2008), «Indios y ladinos, arraigados y migrantes en Chiapas: un esbozo de historia demográfica de larga duración», en Daniel Villafuerte y María del Carmen García (coords.), Migraciones en el sur de México y Centroamérica, México, unicach-Miguel Ángel Porrúa Editor.

VILlAFUERTE, Daniel (2001), Integraciones comerciales en la frontera sur, México, ProImMSE-UNAM.

(coord.) (2000), El café en la frontera sur. La producción y los productores del Soconusco, Chiapas, Tuxtla Gutiérrez, Universidad de Ciencias y Artes de Chiapas. , María del Carmen García y Salvador Meza (1997), La cuestión ganadera y la deforestación. Viejos y nuevos problemas en el trópico y Chiapas, Tuxtla Gutiérrez, Universidad de Ciencias y Artes de Chiapas.

Wasserstrom, Robert (1980), Ingreso y trabajo rural en los Altos de Chiapas. Informe final del proyecto «Minifundismo y trabajo asalariado. Estudio de caso II: San Juan Chamula» 1975-1977. Ed. Centro de Investigaciones del Sureste (CIES), Serie Documentos 6, San Cristóbal de Las Casas. 
